\title{
Trichosporon Asahii Infection Mortality in Pediatric Oncology Patients: A Report of Six Cases
}

\author{
Adil Abdelhamed Abbas ${ }^{1}$, Abdulfattah Wasel Alamri ${ }^{2}$, Areej Hassan Almofti ${ }^{2}$ \\ ${ }^{1}$ College of Medicine, King Saud Bin Abdulaziz University for Health Sciences, Pediatric Hematology/Oncology \\ Section, Princess Nourah Oncology Centre, King Abdulaziz Medical City, Jeddah, Saudi Arabia \\ ${ }^{2}$ College of Medicine, King Saud Bin Abdulaziz University for Health Sciences, Department of Laboratory Medicine, \\ King Abdulaziz Medical City, Jeddah, Saudi Arabia
}

\begin{abstract}
The frequency of invasive fungal infections in patients with hematological malignancies has dramatically increased over the last two decades mainly due to increased use of more intensive therapies. Trichosporon spp is an uncommon but frequently fatal invasive yeast infection that occur predominantly in patients with hematological malignancies. Trichosporon Asahii (T. Asahii) is the most common genus isolated and is reported to cause serious invasive infections in adults and infrequently in children. Voriconazole (VCZ) remains the antifungal drug of choice. We report six pediatric patients with acute lymphoblastic leukemia (2), acute myeloblastic leukemia (2), lymphoblastic lymphoma (1) who developed invasive T. Asahii infection during the early phases of CTR. The outcome was poor. Five patients have died as a result of the infection after a stormy clinical course and prolonged stay in the pediatric intensive care unit despite receiving proper antifungal treatment. Only one patient survived the infection without serious sequalae. J Microbiol Infect Dis 2020; 8(3):167-172.
\end{abstract}

Keywords: Amphotericin B, Caspofungin, Invasive fungal infections, Trichosporon Asahii, Voriconazole

\section{INTRODUCTION}

The incidence of invasive fungal infections in patients with hematological malignancies has dramatically increased over the last two decades, mainly as a result of the increased use of intensive combination cytotoxic regimens, increased use of allogeneic BMT, and the recently increased use targeted and immune modulating therapy. Various groups have stressed on the importance of new opportunistic fungi as causes of life-threatening infections. The pathogens most commonly implicated in invasive Trichosporon (TS) infection are the yeasts widely referred to as T. sp. and Geotrichum capitatum [1,2].

TS infection is an uncommon but frequently fatal invasive fungal infection in immunocompromised patients, particularly those with hematological malignancies. The genus TS contains 51 species, 16 of which are able to infect human hosts. T. sp. infection has frequently been reported in immunocompromised children especially those with hematological malignancies, chronic respiratory diseases and autoimmune cholangitis [3-6]. Here we report six children with malignancy that developed invasive T. Asahii infection and were treated in our hospital over the last six years. The outcome was poor as five of those patients died as a result of the infection. In this report we discuss the epidemiology, pathogenesis, clinical presentation and the use of antifungal therapy for treatment of invasive T. Asahii infection.

\section{CASE REPORT}

\section{Case 1}

A 3-year old girl was diagnosed with standard risk ALL and was treated with CTR according to the CALL08 protocol (https://www.kfshrc.edu.sa/store/media/1gx.pdf). Starting from the sixth day of induction CTR (dexamethasone, vincristine, L. asparaginae), she developed neutropenic fever and was 
started on broad spectrum antibiotics. Caspofungin (CFN) was subsequently added for persistent fever. Three successive blood cultures obtained from the Percutaneously Inserted Central Catheter (PICC) grow T. Asahii, sensitive to amphotericin $B(A m B)$ and VCZ. CFN was then stopped and AmB lipid complex (abelcet) was started. Computerized Tomography (CT) scan of the lungs showed tree on bud appearance suggestive of invasive fungal disease. CT scan of the abdomen showed fungal infiltration of the spleen and kidneys (Figure $1 \mathrm{a} \& \mathrm{~b}$ ).

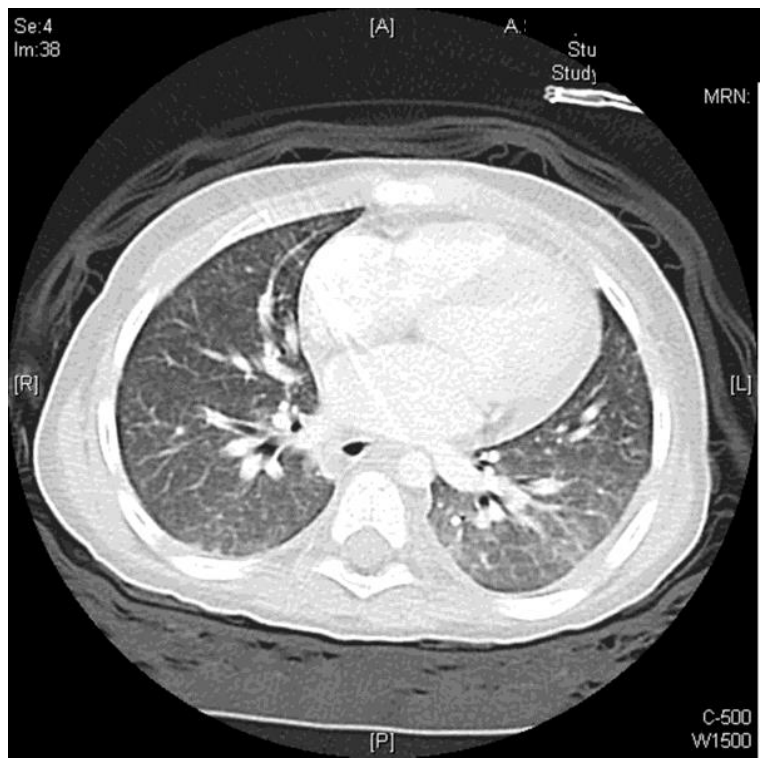

Figure $1 \mathrm{a} \& \mathrm{~b}$ : CT scan of the chest; (a) abdomen and pelvis (b) with contrast (sagittal view): showing alveolo - bronchial inflammatory changes in the lung bases and left upper lung lobe with scattered classic tree on bud nodules and a small left side pleural effusion. Mildly enlarged liver. Spleen and kidneys are not enlarged but contained multiple innumerable tiny hypodense nodules most probably representing fungal infection.

Fever and neutropenia persisted but the blood cultures remained negative. VCZ was subsequently added. A papules of red colored skin rash appeared and quickly spread all over the upper part of her body (Figure 2). A punch skin biopsy showed fungal elements. She remained febrile and neutropenic. She suddenly deteriorated with hypotension and required inotrobes and mechanical ventilation. Unfortunately, she died few days later after repeated cardiac arrests.

\section{Case 2}

A 13 years old boy with newly diagnosed AML, M1, was treated according to the CAML15 protocol. Following his first cycle of ADE (Ara C, daunorubicin, etoposide) CTR, he developed prolonged neutropenic fever and was treated with combination empiric antibiotics and CFN. He suddenly developed septic shock and was transferred to the PICU. Blood culture obtained from the PICC line grew T. Asahii and so did the catheter tip after removal. CT scan showed disseminated fungal infection affecting the lungs and the liver (Figure 3). The blood cultures remained positive for $T$. Asahii despite the use of Abelcet and VCZ to which the organism was fully sensitive. He progressed to develop severe respiratory distress and required mechanical ventilation. He unfortunately died nearly 3 weeks later due to respiratory failure.

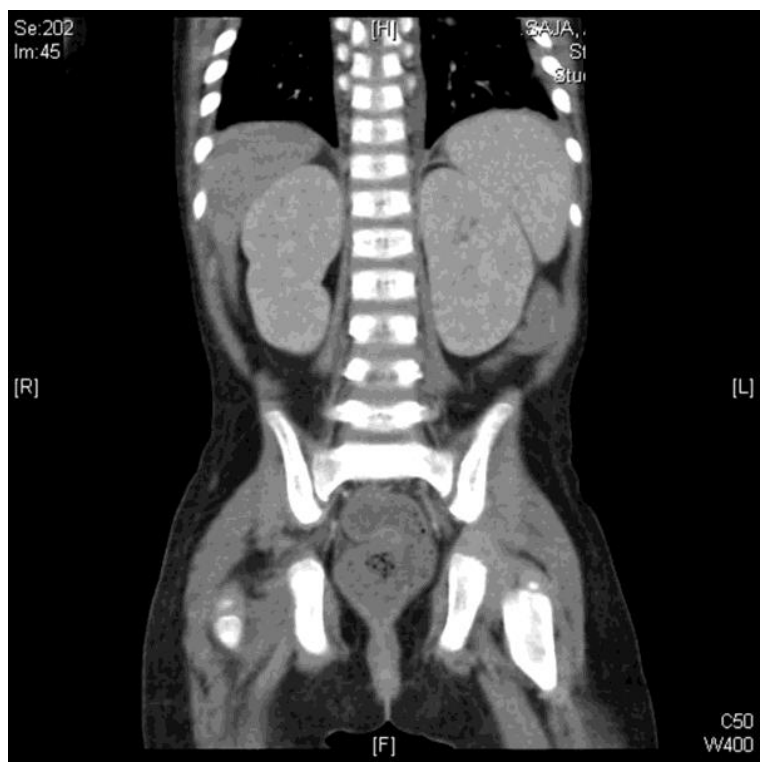

Figure 2. The patient while mechanically ventilated in intensive care unit. Note the presence of the scattered maculo - popular reddish fungal skin rash of almost the same size $(3-4 \mathrm{~mm})$ affecting the neck, chest, abdomen and upper limbs.

\section{Case 3}

A 13 years old girl was diagnosed with AML, M5 and was treated according to the CAML09 protocol. Immediately following her first cycle of ADE CTR, she developed prolonged neutropenic fever and was transferred to the PICU for culture negative septic shock. Right after arrival to the PICU, she developed cardiac arrest for nearly 10 minutes but responded to resuscitation. Unfortunately, the brain CT and MRI scans following resuscitation showed 
widespread ischemic changes. While in PICU and receiving empiric broad spectrum antibiotics and CFN, her blood culture obtained from the femoral catheter grew T. Asahii. The catheter was removed, CFN stopped and abelcet and VCZ to which the organism was sensitive were started. CT scan examination showed multiple splenic hypodense lesions suggestive of fungal infection. The blood culture continued to be positive for the next 2 days. She deteriorated and developed disseminated intravascular coagulation with bleeding from multiple body sites (nose, moth and rectum). Unfortunately, she died few days later because of circulatory failure.

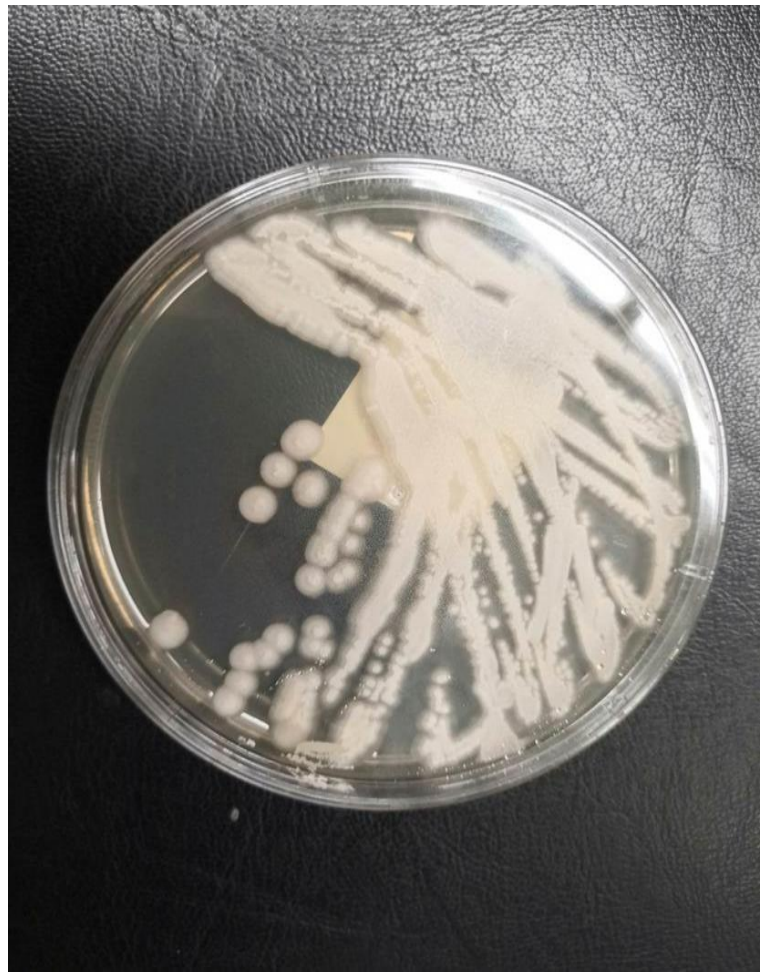

Figure 3. Heavy growth of T. Asahii on Sabouraud dextrose agar. Identification and antifungal sensitivity were subsequently performed using the Vitec -MS and the Vitec -II identification and sensitivity systems.

\section{Case 4}

A 9-year old boy with relapsed persistent mediastinal precursor T- cell LL was admitted to the hospital for neutropenic fever and oral thrush. He had just finished his first cycle of the fourth line CTR (nelarabine, cyclophosphamide and etoposide). His tumor had clearly progressed following CTR. He remained febrile and pancytopenic for nearly 4 weeks and received multiple combinations of broad- spectrum antibiotics and empiric CFN. His blood culture initially grew coagulase negative staphylococci but the subsequent blood cultures obtained from the porta - cath grew T. Asahii in 2 successive days. CT scan showed no evidence of invasive fungal infection. He was transferred to the PICU and received full support but he gradually deteriorated despite being on Abelcet and VCZ in addition to the broad spectrum antibiotics. Unfortunately, he died days later due to cardiorespiratory arrest.

\section{Case 5}

A 4 years old boy was diagnosed with high - risk precursor $B A L L$ and was treated according to the CALL09 protocol. He responded relatively well initially but suddenly presented with progressive thrombocytopenia and was diagnosed with high - risk bone marrow (BM) relapse at week \#75 of maintenance CTR. He was treated with CTR according to a the local CRALL09 relapse protocol. He received week \#1 and \#2 CTR (Dexamethasone, vincristine, L. asparaginase and idarubicin) and went severely neutropenic. He developed repeated high spikes of temperature and became very sick. He was treated with broad spectrum antibiotics and empiric CFN. His blood cultures obtained from the porta - cath grew T. Asahii in five successive days. CT scan showed no evidence of invasive fungal disease. Antifungal cover was changed to VCZ and abelcet however, he continued to deteriorate. Unfortunately, he lost his battle with the disease and died few days later due to circulatory failure.

\section{Case 6}

A 3 years old girl with AML, M5 was treated according to the CAML15 protocol. Following the first induction cycle of CTR (ADE), she developed prolonged fever and neutropenia and was placed on broad spectrum antibiotics and CFN. Blood cultures obtained from the porta cath grew T. Asahii in three successive days. CT scan showed few hypodense lesions in the spleen and kidneys suggestive of invasive fungal infection. CFN was then replaced with VCZ to which the organism was sensitive. With the recovery of the neutrophil counts fever subsided and the blood cultures became negative. She became well and was continued on VCZ for the whole duration of therapy. 
Repeat CT scan following treatment completion showed resolution of all fungal lesions.

\section{DISCUSSION}

Between December 2012 and October 2018, six of our pediatric oncology patients were diagnosed with invasive fungal infection due to T. Asahii. Unfortunately, five of these patients suffered severe morbidity due to the infection and required intensive management in the PICU, before they died as a result of the infection. As much as five of our six patients developed the infection early during their intensive CTR course. In fact, out of the five patients who died, three patients were newly diagnosed with leukemia who received only the first part of the induction CTR, one patient had relapse ALL and one patient had relapsed LL. The last two patients had just started on the intensive reinduction course of CTR when they developed the infection, indicating the significance of seriously considering the possibility of $T$. Asahii infection and starting prompt treatment from the outset.

Despite the increasing attention being focused on all fungal infections, little is known about the current epidemiology of these emerging pathogens. T. sp. are basidiomycetous yeastlike small organisms. They are widely distributed in nature and can generally be isolated from soil, water, decomposing matter, and bird and bat droppings. T. sp. constitute part of the transient human microbiota, mainly in the skin, nails and the mucosa of the respiratory and gastrointestinal tracts [5-8].

T sp. are best cultured on blood, chocolate and Sabarod agars. Final identification of is performed by sequencing the ribosomal DNA (rDNA) intergenic spacer 1 (IGS1) using modern technology such as the Vitek-MS system. This test is considered the standard and is currently mandatory for accurate identification of $\mathrm{T}$. sp. [913].

T. sp. has now become an important fungal pathogen in patients with hematological malignancies. The number of cases reported have increased over the past decade, $T$ sp. has become the second most common yeast genus causing fungemia in such patients after Candida sp [14-16].
The most common predisposing conditions for invasive TS infection are hematological malignancies especially acute leukemia [17]. Liao $Y$ et al reviewed 185 cases of TS fungemia reported between 1975 and 2014. The frequency of case repots increased progressively with time. Cases were reported in four continents (North America, Europe, Asia and South America). The mean age at presentation was 47 years (ranging from 0 to 84). Most cases were males (66.12\%),. Hematological diseases especially acute leukemia accounted for most patients (36\%). Most patients had a history of neutropenia (60.49\%), CTR treatment (58.44\%), antimicrobial use $(84.05 \%)$, prophylactic/empirical antifungal therapy $(43.24 \%)$, or central venous catheter (CVC) use $(52.81 \%)$ before or at the onset of the infection. A total of $185 \mathrm{~T}$. sp. strains were isolated from blood samples and 76 isolates were classified at the species level. The most commonly isolated TS species was T. Asahii (75\%). A total of 109 patients $(58.92 \%)$ experienced a negative outcome (death/worsening), and 76 patients $(41.08 \%)$ experienced a positive outcome (cure/improvement). In a univariate analysis, a significantly greater proportion of patients with a negative outcome had a history of using antimicrobials, prophylactic/empirical antifungal therapy, or bacterial bloodstream coinfection $[10,15]$.

Invasive TS fungemia is a life-threatening infection that was almost exclusively seen in neutropenic adults with acute leukemia and was associated with poor prognosis. The disease is uncommon in children [18]. Few pediatric cases were reported in the literature with variable treatment outcomes. Maxfield L. et al, reported a 3- years old girl with ALL who developed fatal $T$. Asahii fungaemia after induction CTR while on micfungin treatment for prolonged fever and neutropenia. She was initially treated with VCZ but on disease progression with respiratory failure and mechanical ventilation due to sepsis, liposomal amphotericin B (LAmB) was added. However, she continued to deteriorate to ultimately died at day 105 of admission [19]. In contrast, Tanyildiz HG. et .al, reported two pediatric patients; a 2-years old boy, with extensive Langerhans cell histiocytosis and a 12 years old boy with AML. They developed 
invasive T. Asahii fungaemia with several positive blood cultures. Both patients responded well to treatment with VCZ [16].

$A m B$ and $L A m B$ were the most frequently used monotherapies during the 1970's, 1980's and 1990's. Therapy with a single triazole (VCZ, fluconazole, itroconazole and miconazole) has increased to become the most frequently used drug during 2005-2014. In general, fungicidal activity of AmB against. Currently, triazole especially VCZ are the preferred drugs for the treatment [18]. A significant proportion of patients experiencing a negative outcome received the antifungal AmB. However, a significantly greater proportion of patients experiencing a positive outcome received an antifungal regimen using $\mathrm{VCZ}$ or a regimen using an AmB-triazole combination [15]. This variability in the response to antifungals and the ultimate outcome may be explained by partial resistance of the organism to antifungals as some isolates may have high minimum inhibitory concentration (MIC) and /or the ability to form biofilms within the body tissues or the lumen of the CVC's. Therefore, such patient continues to shed organisms into the circulation and the blood culture becomes positive for several days to weeks despite optimal antifungal therapy [5].

All the six pediatric patients who developed invasive TS infection in our hospital had several risk factors for the development of infection in common; malignant disease (all patients had hematological malignancies), intensive CTR regimen, prolonged neutropenia, empiric antibiotic and antifungal therapies, and the presence of CVC's. They were all treated with either AmB, abelcet, VCZ, miconazole or a combination of two antifungals. The organism was universally sensitive to AmB and VCZ. However, and despite the similarities, the outcomes were very different ranging from complete cure without sequalae to death in the vast majority of patients.

For example, our first patient had standard risk ALL. During her second week of induction, she developed fever and remained neutropenic. She had persistently high fever for more than 3 weeks. The blood cultures obtained initially from the CVC and subsequently from peripheral blood were continuously positive for $T$. Asahii for more than one week. Biopsy from the skin lesion showed fungal elements but fungi did not grow in culture medium. CT scan images showed fungal dissemination into the lungs, kidneys and spleen. The organism isolated was sensitive to both VCZ and Abelcet, which she received in combination. However, she progressed to full septicemia, dropped her blood pressure and required inotropic support and mechanical ventilation. She died few days later after several resuscitation attempt for cardiac arrests. Our sixth patient however, was diagnosed with AML. She developed neutropenic fever following her first induction course of CTR. Two blood cultures obtained from the CVC were positive for $T$. Asahii. CT scan images showed dissemination into the kidneys and spleen. She was treated with VCZ and in contrast to the first patient, she responded very well and cleared her infection.

We are not sure what made that big difference in the outcome between our patients, as nearly all patient had similar clinical presentation and preexisting risk factors for the development of infection. Further studies are for sure needed to elucidate the exact pathogenesis of $T$. Asahii infection and to determine other factors that may be associated with poor outcome. When there is high index of suspicion, the use of combination antifungals with LAmB and VCZ from the start may be justified. For now, and overall, increased awareness of this emerging rare but fatal fungal infection is very important as early suspicion of the infection and early start of the appropriate antifungal therapy may help to combat the infection and prevent severe morbidity and mortality.

\section{ACKNOWLEDGMENTS}

Conflict of interest: The authors declare no personal or financial conflict of interest.

Financial Disclosure: No financial support was received

\section{REFERENCES}

1- Anaissie, E. J. Opportunistic mycoses in the immunocompromised host: experience at a cancer center and review. Clin. Infect. Dis. 1992, 14 (Suppl 1):43 - 53 .

2- Pfaller, M. A. Epidemiology and control of fungal infections. Clin. Infect. Dis.1994, 19 (Suppl 1): S8 S13.

3- Thibeault R, Champagne M, de Repentigny L, et. al. Fatal disseminated Trichosporon Asahii infection in a child with acute lymphoblastic 
leukemia. Can J Infect Dis Med Microbiol 2008; 19 (2): 203 - 205.

4- Kröner C, Kappler M, Grimmelt AC, et al. The basidiomycetous yeast Trichosporon may cause severe lung exacerbation in cystic fibrosis patients clinical analysis of Trichosporon positive patients in a Munich cohort. Pulmonary Medicine 2013, 13; 61: $2-10$.

5- Iturrieta - Gonzalez IA, Padovan AB, Bizerra1 FC, Hahn RC, Colombo AL. Multiple Species of Trichosporon Produce Biofilms Highly Resistant to Triazoles and Amphotericin B. PLoS One. 2014 Oct 31; 9 (10): e109553. doi: 10.1371/journal.pone. 0109553. eCollection 2014.

6- Noailly Charny PA, Rohmer B, CollardeauFrachon S, Gillet Y. Invasive Hepatobiliary Trichosporon Asahii Infection in a Child with Autoimmune Cholangitis. J Pediatric Infect Dis Soc. 2019 May 20.

7- Gueho, E, de Hoog GS, Smith MT. Neotypification of the genus Trichosporon. Antonie Leeuwenhoek 1992, 61:285-288.

8- Gueho, E., Improvisi L, de Hoog GS, Dupont B. Trichosporon on humans: a practical account. Mycoses 1994, 37: 3 - 10.

9- de Hoog, G. S., Smith MT, Gue'ho E. A revision of the genus Geotrichum and its teleomorphs. Stud. Mycol. 1986, 29:1 - 131.

10- Gue'ho, E., de Hoog GS, Smith MT, Meyer SA. DNA relatedness, taxonomy, and medical significance of Geotrichum capitatum. J. Clin. Microbiol. 1987, 25:1191-1194.

11- Smith, MT, Poot GA. Dipodascus capitatus, Dipodascus spicifer and Geotrichum clavatum: genomic characterization. Antonie Leeuwenhoek 1998, 74: 229 - 235.

12- Salkin, IF, Gordon MA, Samsonoff WA, Rieder CL. Blastoschizomyces capitatus, a new combination. Mycotaxon 1985, 22: 375 - 380.

13- Chagas-Neto TC, Chaves GM, Melo SA, Colombo AL. Bloodstream Infections Due to Trichosporon spp.: Species Distribution, Trichosporon Asahii Genotypes Determined on the Basis of Ribosomal DNA Intergenic Spacer 1 Sequencing, and Antifungal Susceptibility Testing. J Clin Microbiol 2009; 47 (4): 1074-1081.

14- Girmenia C, Pagano L, Martino P, et al. Invasive Infections Caused by Trichosporon Species and Geotrichum capitatum in Patients with Hematological Malignancies: a Retrospective Multicenter Study from Italy and Review of the Literature. J Clin Microbiol, 2005, 43 (4): 1818 1828.

15- Liao Y, Lu X, Yang S, Luo Y, Chen Q, Yang R. Epidemiology and Outcome of Trichosporon Fungemia: A Review of 185 Reported Cases From
1975 to 2014. Open Forum Infect Dis. 2015 Sep 25; $2(4): 1-10$.

16- Tanyildiz HG, Yesil S, Toprak S, Candir MO, Sahin G. Two Case Presentations Infected by Trichosporon Asahii and Treated with Voriconazole Successfully. Case Rep Infect Dis. 2015; 2015: 651315. doi: 10.1155/2015/651315. Epub 2015 Aug 13.

17- Suzuki K, Nakase K, Kyo T, et al. Fatal Trichosporon fungemia in patients with hematologic malignancies. Eur J Haematol 2010; 84: 441 - 7.

18- Hazırolan G. An overview on Trichosporon Asahii and its infections. Mikrobiyol Bul. 2012 Oct; 46 (4): 707 - 15.

19- Maxfield L, Matthews JJ, Ambrosetti DR, Ephtimios IE. Trichosporon fungemia in a pediatric patient with acute lymphoblastic leukemia. ID Cases 2 (2015) $106-108$ 